EPJ manuscript No.

(will be inserted by the editor)

\title{
Bond dilution in the 3D Ising model: a Monte Carlo study
}

\author{
Pierre-Emmanuel Berche ${ }^{1 \mathrm{a}}$, Christophe Chatelain ${ }^{2}$, Bertrand Berche ${ }^{2}$ and Wolfhard Janke ${ }^{3}$ \\ 1 Groupe de Physique des Matériaux (UMR CNRS No 6634), Université de Rouen, \\ F-76801 Saint Etienne du Rouvray Cedex, France \\ ${ }^{2}$ Laboratoire de Physique des Matériaux (UMR CNRS No 7556), Université Henri Poincaré, Nancy 1 , \\ F-54506 Vandœuvre les Nancy Cedex, France \\ 3 Institut für Theoretische Physik, Universität Leipzig, Augustusplatz 10/11, \\ D-04109 Leipzig, Germany
}

September 2, 2018

\begin{abstract}
We study by Monte Carlo simulations the influence of bond dilution on the three-dimensional Ising model. This paradigmatic model in its pure version displays a second-order phase transition with a positive specific heat critical exponent $\alpha$. According to the Harris criterion disorder should hence lead to a new fixed point characterized by new critical exponents. We have determined the phase diagram of the diluted model, between the pure model limit and the percolation threshold. For the estimation of critical exponents, we have first performed a finite-size scaling study, where we concentrated on three different dilutions. We emphasize in this work the great influence of the cross-over phenomena between the pure, disorder and percolation fixed points which lead to effective critical exponents dependent on the concentration. In a second set of simulations, the temperature behaviour of physical quantities has been studied in order to characterize the disorder fixed point more accurately. In particular this allowed us to estimate ratios of some critical amplitudes. In accord with previous observations for other models this provides stronger evidence for the existence of the disorder fixed point since the amplitude ratios are more sensitive to the universality class than the critical exponents. Moreover, the question of non-self-averaging at the disorder fixed point is investigated and compared with recent results for the bond-diluted $q=4$ Potts model. Overall our numerical results provide evidence that, as expected on theoretical grounds, the critical behaviour of the bond-diluted model is governed by the same universality class as the site-diluted model.
\end{abstract}

Key words. Ising model - disorder - bond dilution - Monte Carlo simulation

PACS. 05.40.+j Fluctuation phenomena, random processes, and Brownian motion - 64.60.Fr Equilibrium properties near critical points, critical exponents - 75.10.Hk Classical spin models

\section{Introduction}

The influence of quenched, random disorder on phase transitions has been the subject of numerous experimental and theoretical investigations since more than 20 years [1]. They concern both first- and second-order phase transitions, especially in two dimensions. The qualitative influence of quenched, short-range correlated [2] random disorder at second-order phase transitions is well understood since Harris 3] proposed a relevance criterion based on the knowledge of the specific heat critical exponent $\alpha$ of the pure model: when $\alpha$ is positive, under a coarse graining, the disordered system should reach a so-called finiterandomness disorder fixed point characterized by altered critical exponents, whereas if $\alpha$ is negative, the universality class of the pure system will persist. In particular

\footnotetext{
a Author for correspondence (pierre.berche@univ-rouen.fr).
}

the two-dimensional (2D) Ising model has attracted great interest in the past years because of its intermediate situation $(\alpha=0)$ which implies a marginal influence of disorder 4]. From an experimental point of view, a confirmation of the Harris criterion was reported in a LEED investigation of a 2D order-disorder transition [5, 6]. In the case of a first-order phase transition in the pure model, disorder is expected to soften the transition and, under some circumstances, may even induce a second-order transition [7. For 2D systems, the latter scenario has been proved by Aizenman and Wehr on rigorous theoretical grounds 8 , 9. To test these theoretical predictions also the Potts model 10 has been intensively studied in 2D [1] since it displays the two different regimes: a second-order phase transition when the number of states per spin $q \leq 4$ and a first-order one when $q>4$. These results were obtained by different techniques including Monte Carlo simulations, transfer matrix calculations, field-theoretic perturbation 
P.E. Berche, C. Chatelain, B. Berche and W. Janke: Bond dilution in the 3D Ising model

theory, and high-temperature series expansions [12,13. 14, 15, 16, $17,18,19,20,21,22$.

In three dimensions (3D), the disordered Potts model has of course been studied only later: the case $q=3$, corresponding to a very weak first-order transition for the pure model 23], has been investigated numerically by Ballesteros et al. 24 for site dilution, and the case $q=4$, exhibiting in the pure system a strong first-order transition [25], has been studied very recently by us [26, 27. 28 29 30 for bond dilution via large-scale Monte Carlo simulations. Only the 3D Ising model with site dilution has also been extensively studied using Monte Carlo sim\begin{tabular}{l|l|l|l|l|l|l|l|l|l|l|l|l|} 
ulations & 31 & 32,33 & 34 & 35 & 36 & 37 & 38,39 & 40 & 41,42 & 43 & 44,45,
\end{tabular} or field theoretic renormalization group approaches 46 , 47, 48, 49,50,51,52,53,54,55. The diluted model can be treated in the low-dilution regime (concentration of magnetic bonds, $p$, close to 1 ) by analytical perturbative renormalization group methods $56,57,58,59,60$ where a new fixed point independent of the dilution has been found, but for stronger disorder only Monte Carlo simulations remain valid.

The first numerical studies 32,33 , 34 suggested a continuous variation of the critical exponents along the critical line but after the works 35, 36, 37,38,39 it became clear that the concentration dependent critical exponents found in Monte Carlo simulations are effective ones, characterizing the approach to the asymptotic regime. The critical exponents $\beta$ and $\gamma$ associated with the magnetisation and susceptibility, respectively, were shown by Heuer 38 to be concentration dependent in the region $0.5 \leq p<1$. The conclusion of Heuer was: while a crossover between the pure and weakly random fixed points accounts for the behaviour of systems above $p \simeq 0.8$, in more strongly disordered systems a more refined analysis is needed. We should mention here that the meaning of $p$ is not exactly the same in the papers mentioned above which refer to site dilution and in our present study in which we are interested in bond dilution. That is why the numerical values of $p$ given before for the different regimes cannot directly be taken over to the bond-dilution case.

Another important question has been investigated by Wiseman and Domany 43,44: it concerns the question of the possible lack of self-averaging which can happen in disordered systems. For the 3D site-diluted Ising model close to criticality they explicitly showed that physical quantities such as the magnetisation or susceptibility are indeed not self-averaging. Although simulations 44 revealed that disorder realized in a canonical manner (fixing the fraction $p$ of magnetic sites) leads to different results than those obtained from disorder realized in a grandcanonical ensemble (assigning to each site a magnetic moment with probability $p$ ), the renormalization-group approach of Ref. 61 shows that the canonical constraint is irrelevant, even near the random fixed point, suggesting that the observed differences are a finite-size effect. The studies of Ref. 42 were based on the crucial observation that it is important to take into account the leading corrections-to-scaling term in the infinite-volume extrapolation of the Monte Carlo data. Thus, the main problem encountered in these studies of the disordered Ising model was the question of measuring effective or asymptotic exponents. Although the change of universality class should happen theoretically for arbitrarily weak disorder, the new critical exponents appear only in a small temperature region around the critical point, whose size is controlled by the concentration of the non-magnetic compound. Equivalently, in finite-size scaling studies very large lattices are required to observe the asymptotic behaviour. In fact, the asymptotic regions cannot always be reached practically and one therefore often measures only effective exponents.

Another crucial problem of the new critical (effective or asymptotic) exponents obtained in these studies is that the ratios $\beta / \nu$ and $\gamma / \nu$ occuring generically in finite-size scaling analyses are almost identical for the disordered and pure models. For the pure 3D Ising model, accurate values are 62:

$$
\nu=0.6304(13), \quad \eta=0.0335(25)
$$

which gives:

$$
\beta / \nu=0.517(3), \quad \gamma / \nu=2-\eta=1.966(3),
$$

and $\alpha=0.1103(1)>0$, i.e., disorder should be relevant according to the Harris criterion.

For the site-diluted model, the asymptotic exponents given by Ballesteros et al. 42] are:

$$
\beta / \nu=0.519(3), \quad \gamma / \nu=1.963(5), \quad \nu=0.6837(53)
$$

Thus, finite-size scaling techniques will only be able to differentiate between the values of $\nu$ at the two fixed points, but will not be very efficient for distinguishing ratios of critical exponents. Even if $\beta$ and $\gamma$ themselves happen to be quite different, the ratios $\beta / \nu$ and $\gamma / \nu$ are very close. That is the reason why a study of the temperature behaviour of the magnetisation and susceptibility will be very helpful for an independent determination of the exponents $\beta$ and $\gamma$.

Contrary to previous studies of the disordered Ising model which were concerned with site dilution, we have chosen to model the disorder by bond dilution in order to compare these two kinds of disorder and to verify that they indeed lead to the same set of new critical exponents, as expected theoretically by universality arguments. In the following we shall thus consider the bond-diluted Ising model in 3D whose Hamiltonian with uncorrelated quenched random interactions can be written (in a Potts model normalization) as

$$
-\beta \mathcal{H}=\sum_{(i, j)} K_{i j} \delta_{\sigma_{i}, \sigma_{j}},
$$

where the spins take the values $\sigma_{i}= \pm 1$ and the sum goes over all nearest-neighbour pairs $(i, j)$. The coupling strengths are allowed to take (grand-canonically) two different values $K_{i j}=K \equiv J / k_{B} T$ and 0 with probabilities 
$p$ and $1-p$, respectively,

$$
\begin{aligned}
\mathcal{P}\left[K_{i j}\right] & =\prod_{(i, j)} P\left(K_{i j}\right) \\
& =\prod_{(i, j)}\left[p \delta\left(K_{i j}-K\right)+(1-p) \delta\left(K_{i j}\right)\right],
\end{aligned}
$$

$c=1-p$ being the concentration of missing bonds, which play the role of the non-magnetic impurities.

The plan of the rest of the paper is as follows: in section 2 , we present the phase diagram of the bond-diluted Ising model. Then, in section 3, we discuss the averaging procedure and investigate the question of possible nonself-averaging of physical quantities. The section 4 is devoted to the critical behaviour of the disordered model. In the first part we present our finite-size scaling study of three particular concentrations $p$, and the second part deals with the temperature scaling for the same three dilutions. Finally, section 5 contains our conclusions.

\section{Phase diagram}

In order to determine the phase diagram and the critical properties at a few selected dilutions we performed in this study large-scale Monte Carlo simulations on simple cubic lattices with $V=L^{3}$ spins (up to $L=96$ ) and periodic boundary conditions in the three space directions, using the Swendsen-Wang cluster algorithm 63. for updating the spins. The histogram reweighting technique 64,65 was employed to extend the results over a range of $K$ around the simulation point. All physical quantities are averaged over 2000 - 5000 disorder realisations, indicated by a bar (e.g., $\bar{m}$ for the magnetisation). Standard definitions were used, e.g., for a given disorder realisation, the magnetisation is defined according to $m=\langle|\mu|\rangle$ where $\langle\ldots\rangle$ stands for the thermal average and $\mu=\left(N_{\uparrow}-N_{\downarrow}\right) /\left(N_{\uparrow}+N_{\downarrow}\right)$. The susceptibility follows from the fluctuation-dissipation relation, $\chi=$ $K V\left(\left\langle\mu^{2}\right\rangle-\langle|\mu|\rangle^{2}\right)$. Here, one should notice that a definition which is usually used in the disordered phase in Monte Carlo simulations, and which seems to be more stable, $\chi=K V\left\langle\mu^{2}\right\rangle$, should be avoided in quenched disordered systems. Simulating at a given temperature above the critical point, one may indeed encounter samples which have higher effective transition temperatures, and which thus have non-zero $\langle|\mu|\rangle$.

The phase diagram was obtained by locating the maxima of the average susceptibility $\bar{\chi}_{L}$ (a diverging quantity in the thermodynamic limit) for increasing lattice sizes $L$ as a function of the coupling strength $K$, with the dilution parameter $p$ varying from the neighbourhood of the pure model $(p=0.95)$ to the very diluted model $(p=0.36)$, see Fig. 1

Below the percolation threshold $p_{c} \simeq 0.2488[66$, one does not expect any finite-temperature phase transition since without any percolating cluster in the system longrange order is impossible. The approximate phase diagram [67] as obtained from the susceptibility maxima for the largest lattice size $(L=20)$ is shown in Fig. 2 For comparison, we have drawn a simple mean-field (MF) estimate of the transition point

$$
K_{c}^{\mathrm{MF}}(p)=p K_{c}(1),
$$

where $K_{c}(1)=0.4433088(6) 68$ is the accurately known transition point of the pure model (in the Potts model normalization), and the single-bond effective-medium (EM) approximation 69

$$
K_{c}^{\mathrm{EM}}(p)=\ln \left[\frac{\left(1-p_{c}\right) e^{K_{c}(1)}-(1-p)}{p-p_{c}}\right],
$$

which gives a very good agreement with the simulated transition line over the full dilution range. We have omitted results from recent high-temperature series expansions 70. since, on the scale of Fig. 2] they would just fall on top of the Monte Carlo data.

To get an accurate determination of $K_{c}(L)$, we used the histogram reweighting technique with at least $N_{\mathrm{MCS}}=$ 2500 Monte Carlo sweeps and between 2000 and 5000 samples of disorder. The number of Monte Carlo sweeps is justified by the increasing behaviour of the energy autocorrelation time $\tau_{e}$ as a function of $p$ and $L$. For each size, we performed at least 250 independent measurements of the physical quantities $\left(N_{\mathrm{MCS}}>250 \tau_{e}\right)$. For a second-order phase transition, the autocorrelation time is expected to behave as $L^{z}$ at the critical point where $z$ is the dynamical critical exponent. For the disordered Ising model, we obtained from the least-squares fits shown in Fig. 3 the values of $z$ compiled in Table 1 We see that the critical slowing-down weakens for the disordered model and that $z$ becomes effectively smaller when the concentration of magnetic bonds $p$ decreases. The observed variation of the dynamical exponent $z$ is probably due to the influence of the different fixed points encountered (pure, disorder and

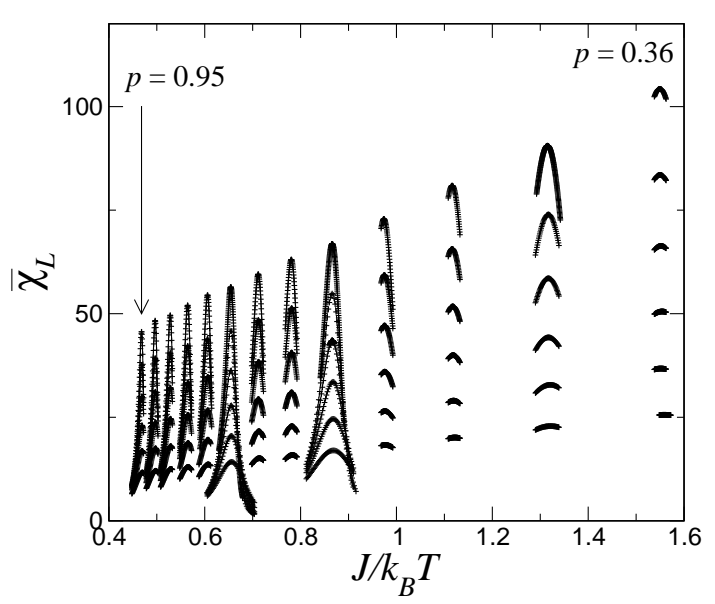

Fig. 1. Variation of the average magnetic susceptibility $\bar{\chi}_{L}$ versus the coupling strength $K=J / k_{B} T$ for several concentrations $p=0.95,0.90, \ldots, 0.36$ and $L=10,12,14,16,18,20$. For each value of $p$ and each lattice size, the curves are obtained by standard histogram reweighting of the simulation data at one value of $K$. 


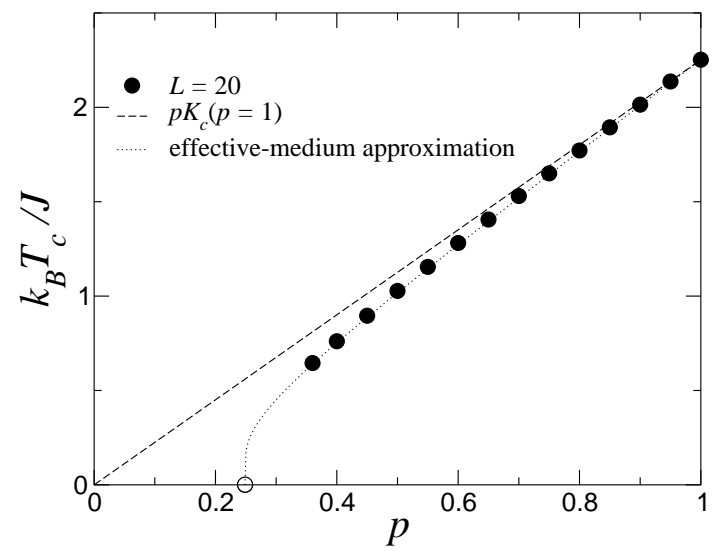

Fig. 2. Phase diagram of the 3D bond-diluted Ising model compared with the mean-field and effective-medium approximations. The open circle marks the location of the percolation threshold.

percolation), as will be discussed below in more detail for the static critical exponents.

The largest autocorrelation time observed for the disordered model was around $\tau_{e} \approx 9$ for $p=0.7$ and $L=96$.

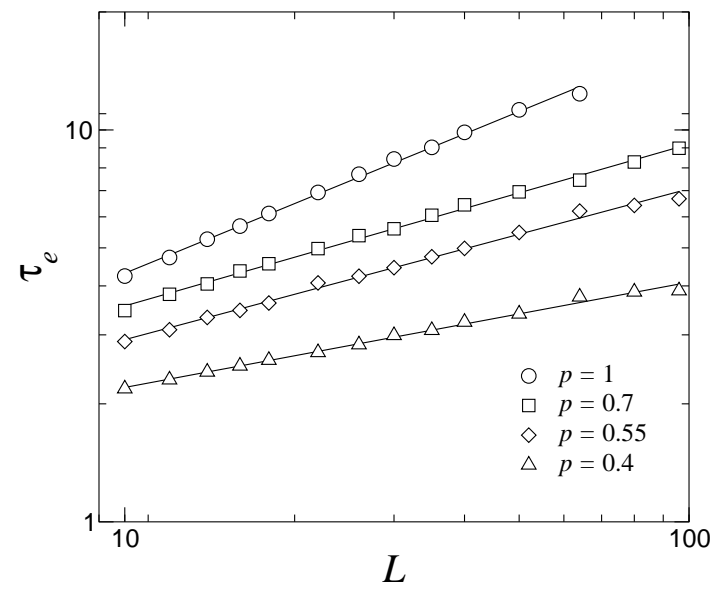

Fig. 3. Energy autocorrelation time $\tau_{e}$ versus the size $L$ of the system on a log-log scale. The pure case corresponds to $p=1$. The straight lines show fits of the form $\tau_{e} \propto L^{z}$, yielding the effective dynamical critical exponents $z$ compiled in Table 1

\section{Non-self-averaging}

In order to achieve accurate results for quenched, disordered systems in numerical simulations it is important to obtain an estimate of the required number of disorder realisations. This is particularly important in the vicinity of a critical point where the correlation length diverges. As a consequence the (disorder) distributions of physical observables typically do not become sharper with increasing system size at a finite-randomness disorder fixed point.
Table 1. Effective dynamical critical exponent $z$ as obtained from linear fits of $\log \tau_{e}$ vs $\log L$. The simulations are performed at the pseudo-critical couplings $K_{c}(L)$ (cf. Table 2 and Fig. [10] below). Here we give in the second line only the simulation coupling $K$ for the largest lattice size $L=96$.

\begin{tabular}{lllll}
\hline$p$ & 1 & 0.7 & 0.55 & 0.4 \\
$K$ & 0.4433 & 0.6535 & 0.8649 & 1.3136 \\
\hline$z$ & 0.59 & 0.41 & 0.38 & 0.27 \\
\hline
\end{tabular}
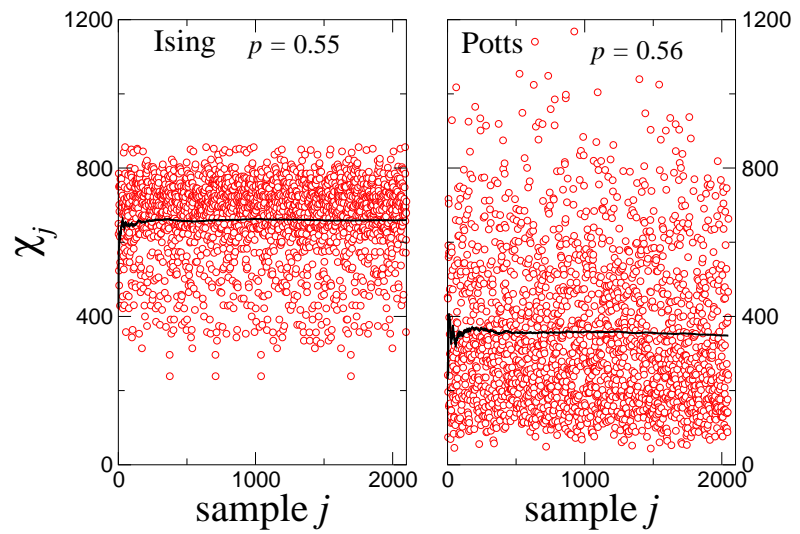

Fig. 4. Disorder distribution of the susceptibility for the Ising and $q=4$ Potts models with a concentration of magnetic bonds of 0.55 and 0.56 , respectively, and a lattice of size $L=64$. The simulations are made at $K=0.8655 \approx K_{c}(L)$ for the Ising model and $K=1.12945 \approx K_{c}(L)$ for the Potts model. In the latter case, the concentration $p=0.56$ belongs to the secondorder regime [26]. The running average over the samples $\bar{\chi}_{j}$ is shown by the thick solid line.

Rather their relative widths stay constant, a phenomenon called non-self-averaging. In order to investigate the disorder averages, we produced $N_{s}$ different samples and computed the corresponding susceptibilities $\chi_{j}, 1 \leq j \leq N_{s}$. In Fig. 4 we compare the distributions for the Ising and $q=4$ Potts models (in the second-order regime [26]). The figure shows that the dispersion of the values of $\chi$ is less important for the disordered Ising model where rare events correspond to low values of $\chi$. This implies that their contribution to the average is not so crucial as for the Potts model where a long rare-event tail is found on the large- $\chi$ side. This is the reason why for the Ising model the fluctuations in the average value disappear after a few hundred realisations and why the disorder averaging procedure is more efficient than for the Potts model.

To test if self-averaging is valid or not for the disordered model [3 44, we investigated the variation of the shape of the probability distributions of the susceptibility when the size is increasing. Dividing the interval $\left(\chi_{\max }-\chi_{\min }\right)$ into 100 bins, we computed the probability for each value of $\chi$ to belong to the bin $i(1 \leq i \leq 100)$. The resulting probability distribution $P\left(\chi_{i} / \bar{\chi}\right)$ (normalized to unity) is drawn in Fig. [5 versus the ratio of the 
average susceptibility $\chi_{i}$ of the bin $i$ and the global average susceptibility $\bar{\chi}$.

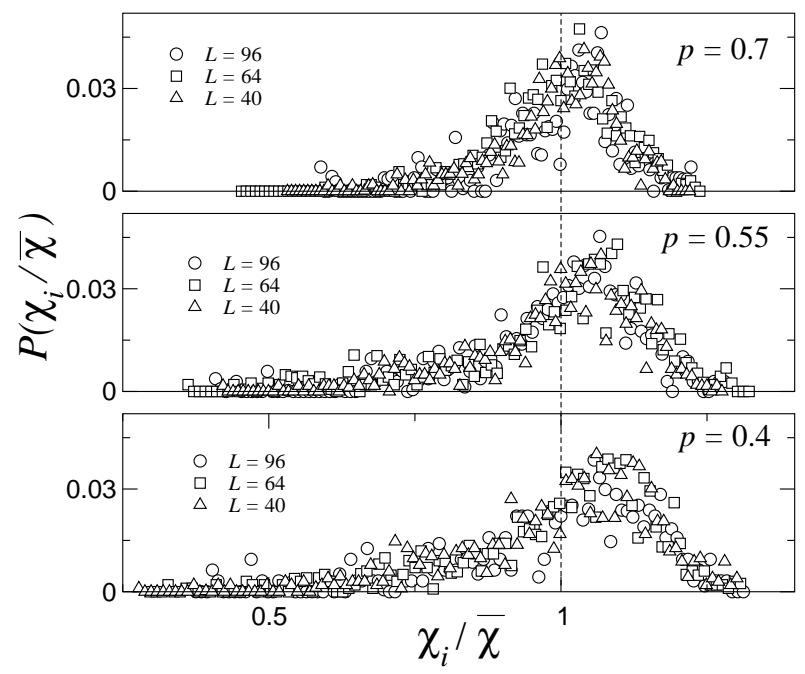

Fig. 5. Probability distribution of the susceptibility versus the ratio of the average value of the susceptibility of bin $i$ and the global average susceptibility $\bar{\chi}$ for the bond-diluted Ising model ( $p=0.7,0.55$, and 0.4 ) for $L=40,64$, and 96. The simulations are performed at $K_{c}(L)$. The vertical dashed line indicates the average susceptibility $\chi_{i} / \bar{\chi}=1$.

For the three studied dilutions, the shape of the curve does not become sharper with increasing size and the general shape remains remarkably independent of the dilution, a phenomenon which strongly contrasts with the situation encountered in the case of the $q=4$ Potts model [26]. Non self-averaging can be quantitatively checked by evaluating the normalized squared width $R_{\chi}(L)=V_{\chi}(L) \overline{\chi(L)}^{2}$, where $V_{\chi}$ is the variance of the susceptibility distribution: $V_{\chi}(L)={\overline{\chi^{2}(L)}}-\overline{\chi(L)}^{2}$. The same quantity is also evaluated for the magnetisation: $R_{m}(L)=V_{m}(L) \overline{m(L)}^{2}$. These ratios are shown versus the inverse lattice size for the three studied concentrations of the disordered Ising model in Fig. 6] The fact that $R_{m}$ and $R_{\chi}$ approach a constant when $L$ increases as predicted by Aharony and Harris 71 is the signature of a non-self-averaging system, in agreement with the results of Wiseman and Domany 43 . 44 for the site-diluted 3D Ising model. In fact, our numerical values of $R_{m}$ are close to the estimate $R_{m}=0.055(2)$ for site dilution reported in Ref. 44]: when the system size goes to infinity, our data are compatible with a convergence towards $R_{m}=0.05(1)$, where the error estimate reflects the uncertainty in the remaining finite-size corrections which appear to be more pronounced than for site dilution. On the other hand, our limiting value for $R_{\chi}$ seems at first sight to be completely off from the estimate $R_{\chi^{\prime}}=0.156$ (4) given in Ref. 44. Upon closer inspection this discrepancy can be traced back to different definitions of the susceptibility. While Wiseman and Domany worked with the "high-temperature" expression $\chi^{\prime}=K V\left\langle\mu^{2}\right\rangle$,

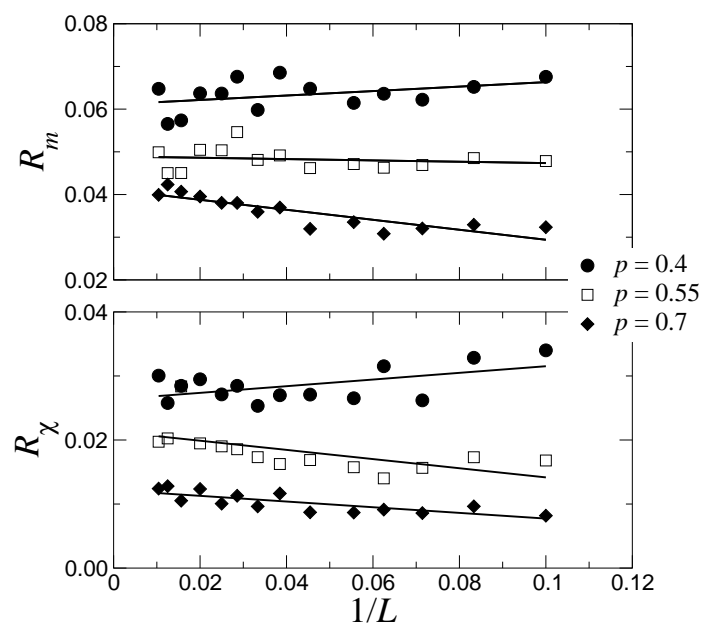

Fig. 6. Normalized squared width of the magnetisation and susceptibility distributions versus the inverse of the lattice size for the three concentrations $p=0.4,0.55$, and 0.7 at the effective critical coupling $K_{c}(L)$. The straight lines are linear fits used as guides to the eye.

we used the connected correlator $\chi=K V\left(\left\langle\mu^{2}\right\rangle-\langle|\mu|\rangle^{2}\right)$, valid in both the low- and high-temperature phase. Another difference is that they evaluated $\chi^{\prime}$ at the infinitevolume critical coupling $K_{c}^{\infty}$ while we followed the pseudotransition points $K_{\max }(L)$ defined from the location of the $\chi$ maxima (see below). In fact, when we evaluate $R_{\chi^{\prime}}$ (i.e., without subtraction), we find for $p=0.7$ and the largest sizes (where $K_{\max }(L) \approx K_{c}^{\infty}$ ) about 10 times larger values than shown in Fig. 6] $R_{\chi^{\prime}}=0.1635,0.1766$, and 0.1630 for $L=64,80$, and 96 . This is in accord with a remark in Ref. 44] who noticed also for site dilution that $R_{\chi}$ is smaller by a factor of $7-10$, which would lead to a crass violation of the leading-order prediction in $\epsilon=4-d$ for the "ratio of ratios" $R_{m} / R_{\chi}=1 / 4$ 71], while working with $\chi^{\prime}$ they obtained a much closer ratio of $R_{m} / R_{\chi^{\prime}}=0.35(2)$. In our $p=0.7$ case we get, with $R_{m}=0.0407,0.0423$, and $R_{m}=0.0399$ for $L=64,80$, and 96, estimates of $R_{m} / R_{\chi^{\prime}}=0.2489,0.2395$, and 0.2448 , in perhaps surprisingly good agreement with the renormalization group prediction of $1 / 4$. Of course, given the large corrections seen otherwise, this agreement may be accidental, but it is also conceivable that the corrections do indeed partially cancel in the "ratio of ratios".

Still, the apparent non-universality of $R_{\chi}$ and to a weaker extent also of $R_{m}$ (the ratios seem to depend on the impurity concentration) remains puzzling. It may be attributed to crossover effects between the pure, disorder and percolation fixed points which may be more sensitive for bond dilution than for site dilution. Another possible reason for quantitative discrepancies might be the fact that $R_{m}$ and $R_{\chi}$ were estimated in our study at the temperature corresponding to the maximum of the average susceptibility for the corresponding lattice size, and not at the critical point of the infinite system. This may lead to different scaling limits (similar to the different Binder parameter scaling limits for pure systems in the vicinity 
Table 2. Values of the simulation points $K=J / k_{B} T$ (extremely close to the susceptibility maxima) for the three mainly studied dilutions.

\begin{tabular}{llll}
\hline$L$ & $p=0.70$ & $p=0.55$ & $p=0.40$ \\
\hline 10 & 0.6560 & 0.8680 & 1.3500 \\
12 & 0.6556 & 0.8680 & 1.3210 \\
14 & 0.6550 & 0.8675 & 1.3185 \\
16 & 0.6546 & 0.8655 & 1.3170 \\
18 & 0.6546 & 0.8655 & 1.3175 \\
22 & 0.6542 & 0.8655 & 1.3160 \\
26 & 0.6541 & 0.8650 & 1.3147 \\
30 & 0.6538 & 0.8650 & 1.3144 \\
35 & 0.6538 & 0.8650 & 1.3142 \\
40 & 0.6538 & 0.8655 & 1.3141 \\
50 & 0.6537 & 0.8653 & 1.3142 \\
64 & 0.6535 & 0.8655 & 1.3144 \\
80 & 0.6535 & 0.8649 & 1.3136 \\
96 & 0.6535 & 0.8649 & 1.3136 \\
\hline
\end{tabular}

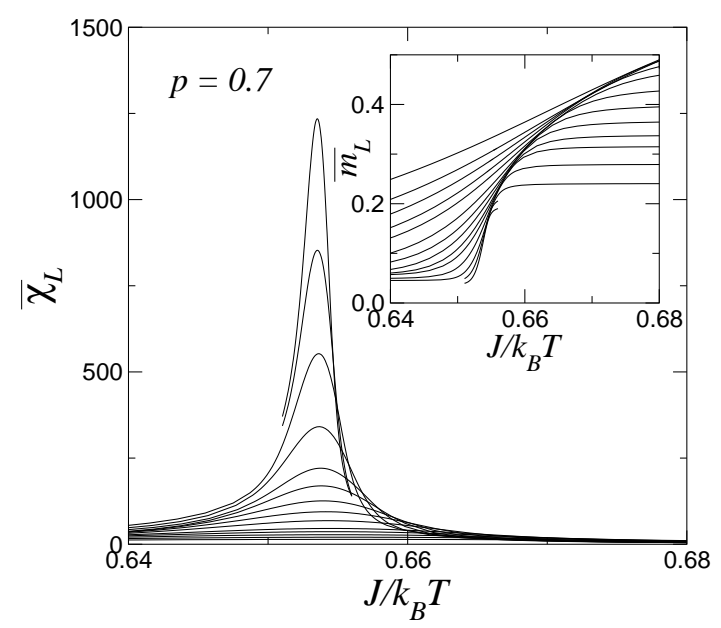

Fig. 7. Variation of the magnetic susceptibility and of the magnetisation (in the insert) versus the coupling strength $K=$ $J / k_{B} T$ for the bond-diluted Ising model $(p=0.7)$ and the lattice sizes compiled in Table 2 going from $10,12,14, \ldots$ to 96. For each value of $L$, the curves are obtained by standard histogram reweighting of the simulation data at the value of $K$ given in Table 2

of $T_{c}$ ), albeit causing presumably only rather small deviations.

\section{Critical behaviour}

In order to study the critical behaviour of the bond-diluted Ising model, we have concentrated on the three particular concentrations $p=0.7,0.55$, and 0.4 , for which the simulated lattice sizes go up to $L=96$. For interested readers, the values of the simulation temperatures are reported in Table 2
In Fig. 7 we show results of typical runs for the magnetisation (in the insert) and the susceptibility for $p=0.7$ at a particular temperature (or coupling) very close to the size-dependent critical point using the histogram reweighting technique. From the scaling of the location and height of the peaks with lattice size of this and similar quantities we then extracted the critical exponents of the system.

\subsection{Finite-Size Scaling Study}

\subsubsection{Correlation length exponent}

As mentioned in the introduction, the vicinity of the exponent ratios $\beta / \nu$ and $\gamma / \nu$ for the pure and disordered universality classes does not allow, by the use of standard finite-size scaling (FSS) techniques, to discriminate between the two fixed points from the behaviour of the magnetisation and the susceptibility, respectively. Only the critical exponent $\nu$, which can be evaluated from the asymptotic FSS behaviour of the derivative of the magnetisation versus temperature,

$$
d \ln \bar{m} / d K \sim a_{d \ln m} L^{1 / \nu},
$$

will be useful with this technique. But even for $\nu$, one is trying to resolve an expected shift from the pure model's value by less than $10 \%$. Taking the data evaluated at $K_{\max }$ and assuming the leading power-law behaviour (5), we have extracted by (linear) two-parameter least-squares fits over successively smaller ranges $L_{\min }-L_{\max }$ the size-dependent effective exponent $(1 / \nu)_{\mathrm{eff}}$. Using the first set of data for lattice sizes $L=4,6,8, \ldots, 20=L_{\max }$, the resulting exponents are plotted in a broad range of concentrations $p$ against $1 / L_{\min }$ in Fig. $8\left(L_{\min }\right.$ is the smallest lattice size used in the fits). We see that in the regime of low dilution ( $p$ close to 1 ), the system is clearly influenced by the pure fixed point. On the other hand, when the bond concentration is small, the vicinity of the percolation fixed point induces a decrease of $1 / \nu$ below its expected disordered value. Indeed, the percolation fixed point is characterized by $1 / \nu \approx 1.1266$.

As is demonstrated in Fig. 9] the same analysis for the three mainly studied dilutions with considerably larger lattice sizes going up to $L=L_{\max }=96$ confirms the observation of the great influence of both the pure and percolation fixed points. The left-most data points in Fig. 9 with $L_{\min }=40$ follow from two-parameter least-squares fits of $(d \ln \bar{m} / d K)_{K_{\max }}$ for our five largest lattice sizes $L=40,50,64,80$, and 96 . Since this leaves only three independent degrees of freedom, moving further into the asymptotic large- $L$ regime would be meaningless with the data available to us. The variation of $(1 / \nu)_{\text {eff }}$ with $L_{\text {min }}$ clearly indicates deviations of the data from the asymptotic ansatz (5), rooted in confluent corrections and crossover terms or both. As will be discussed in more detail below, we found it impossible, however, to resolve these corrections within a non-linear four-parameter ansatz. Still, some rough estimates of the critical exponent $\nu$ can be 


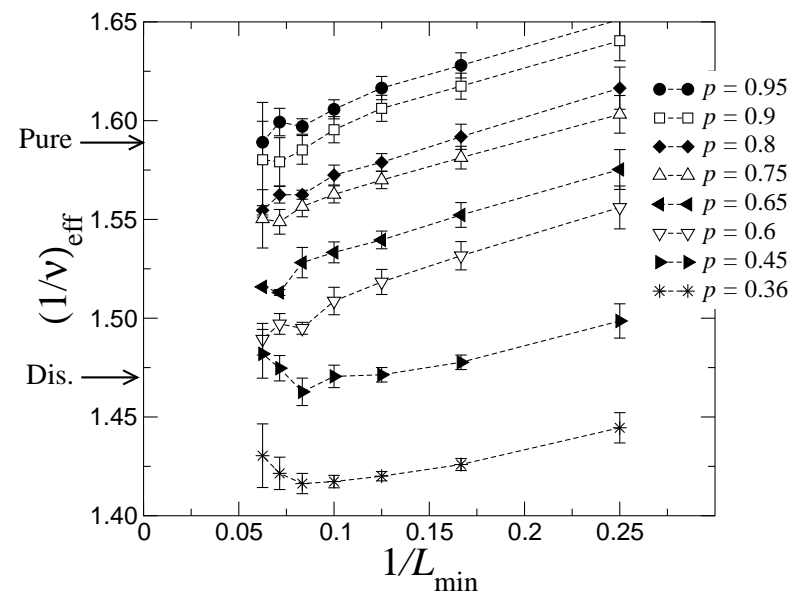

Fig. 8. Effective exponents $(1 / \nu)_{\mathrm{eff}}$ as obtained from powerlaw fits in the range $L_{\min }-L_{\max }=20$ as a function of $1 / L_{\min }$ for $p=0.95,0.9,0.8,0.75,0.65,0.6,0.45$, and 0.36 . The error bars correspond to the standard deviations of the fits. The arrows indicate the values $1 / \nu$ for the pure model [62] and the site-diluted one [42.

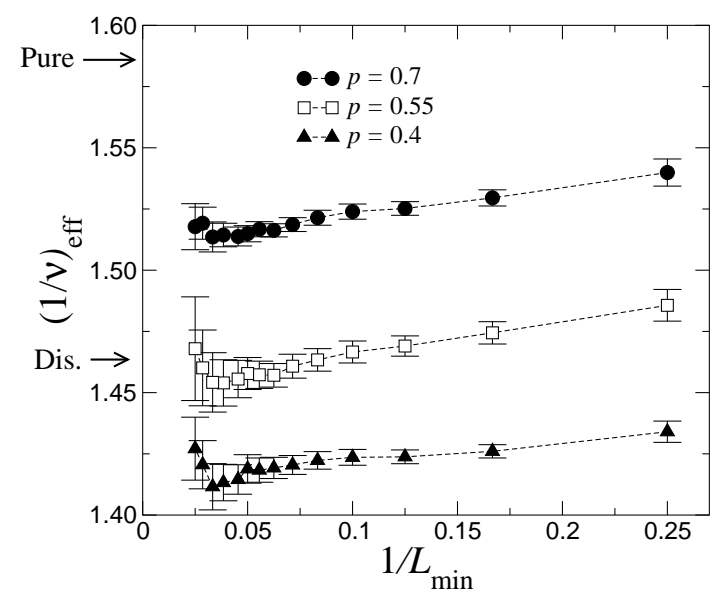

Fig. 9. Effective exponents $(1 / \nu)_{\text {eff }}$ as obtained similar to Fig. 8 from the behaviour of $d \ln \bar{m} / d K$ as a function of $1 / L_{\min }$ for $p=0.7,0.55$, and 0.4 . Here the upper limit of the fit range is $L_{\max }=96$.

read off from Fig. 9 which are collected in Table 3 Obviously, it would be very difficult to extract more precise values from this analysis. At a qualitative level, however, Fig. 9 clearly indicates that the dilution for which the cross-over influence will be the least is around $p=$ 0.55 which suggests that the scaling corrections should be rather small for this specific dilution.

\subsubsection{Critical couplings}

The peak locations of the average susceptibility determine with a good accuracy the size-dependent (pseudo-) critical couplings $K_{c}(L)$, and from an infinite-size extrapolation, $K_{c}(L)=K_{c}^{\infty}+a_{K} L^{-1 / \nu}$, the critical coupling in the thermodynamic limit can be estimated. Inserting the just determined estimate of $\nu$, the linear fit shown in Fig. 10

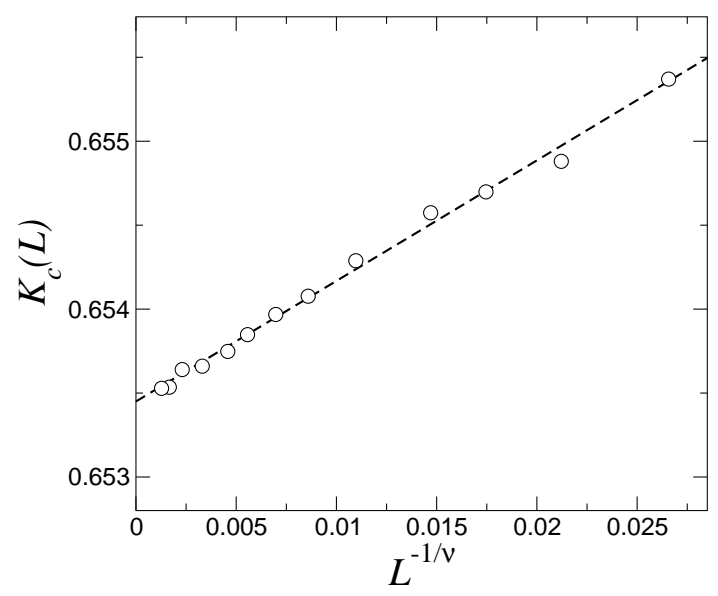

Fig. 10. Size-dependent critical coupling $K_{c}(L)$ versus $L^{-1 / \nu}$ for the bond-diluted Ising model with $p=0.7$, using our estimate of $\nu=0.66(1)$.

for $p=0.7$ yields:

$$
K_{c}^{\infty}=0.6534(1)
$$

The same procedure gives for $p=0.55$ and $p=0.4$ the critical couplings $K_{c}^{\infty}=0.8645(2)$ and $K_{c}^{\infty}=1.3129(3)$, respectively.

\subsubsection{Susceptibility and magnetisation exponents}

As already mentioned, the average magnetisation $\bar{m}$ and susceptibility $\bar{\chi}$ are expected to scale at the size-dependent critical coupling $K_{c}(L)$ with the lattice size as:

$$
\bar{m}_{K_{\max }} \sim a_{m} L^{-\beta / \nu}, \quad \bar{\chi}_{\max } \sim a_{\chi} L^{\gamma / \nu},
$$

where $a_{m}$ and $a_{\chi}$ are non-universal amplitudes. From leastsquares fits to our extensive Monte Carlo data, we computed the effective size-dependent ratios of the critical exponents $(\beta / \nu)_{\text {eff }}$ and $(\gamma / \nu)_{\text {eff }}$ which are plotted in Fig. 11] against $1 / L_{\min }$ for $p=0.7,0.55$, and 0.4 , where $L_{\min }$ is again the smallest lattice size used in the fits.

Concerning the magnetisation, the effective ratios clearly converge, when the size increases, towards $0.515(5)$ for the three dilutions in agreement with the expected values of the pure and site-diluted models. For the susceptibility, on the other hand, the behaviour of $(\gamma / \nu)_{\text {eff }}$ is more differentiated depending on the concentration $p$ : the cases $p=0.7$ and 0.55 are compatible with 1.965(10) if we take into account the error bars, very close to the pure and site-diluted model values, but the case $p=0.4$ displays a $(\gamma / \nu)_{\text {eff }}$ exponent a little bit larger. This discrepancy is probably due to the influence of the percolation fixed point whose $\gamma / \nu$ ratio is close to 2.05 , according to the same scenario as for the exponent $1 / \nu$. The critical exponents from the FSS study are summarized in Table [3 for the three studied dilutions, in good agreement with the hyperscaling relation $d=2 \beta / \nu+\gamma / \nu$.

Following the work of Ballesteros et al. 42] which emphasized the great influence of corrections-to-scaling to get 
Table 3. Critical exponents deduced from the FSS study of the three dilutions.

\begin{tabular}{llll}
\hline$p$ & 0.7 & 0.55 & 0.4 \\
\hline $1 / \nu$ & $1.52(2)$ & $1.46(2)$ & $1.42(2)$ \\
$\nu$ & $0.660(10)$ & $0.685(10)$ & $0.705(10)$ \\
$\beta / \nu$ & $0.515(5)$ & $0.513(5)$ & $0.510(5)$ \\
$\gamma / \nu$ & $1.965(10)$ & $1.977(10)$ & $2.000(10)$ \\
$2 \beta / \nu+\gamma / \nu$ & $2.995(20)$ & $3.003(20)$ & $3.020(20)$ \\
\hline
\end{tabular}

an accurate determination of the critical exponents in the site-diluted Ising model, we tried to estimate them in the bond-diluted version as well. If we consider an irrelevant scaling field $g$ with scaling dimension $y_{g}=-\omega<0$, the scaling expression of, e.g., the susceptibility is:

$$
\bar{\chi}\left(L^{-1}, t, g\right)=L^{\gamma / \nu} f_{\chi}\left(L t^{\nu}, L^{-\omega} g\right) .
$$

At $t=0$, around the fixed point value $g=0$, this leads to the standard expression $a_{\chi} L^{\gamma / \nu}\left[1+b_{\chi} L^{-\omega}+O\left(L^{-2 \omega}\right)\right]$. We hence tried to fit the susceptibility data using the ansatz:

$$
\bar{\chi}_{\max }(L)=a_{\chi} L^{\gamma / \nu}\left(1+b_{\chi} L^{-\omega}\right) .
$$

We systematically varied the exponent ratio $\gamma / \nu$ and the corrections-to-scaling exponent $\omega$ and determined the minimum of $\chi^{2} /$ d.o.f by performing linear fits in the range $18 \leq L \leq 96$ to fix the amplitudes $a_{\chi}$ and $b_{\chi}$. The resulting $\chi^{2}$-landscape for $p=0.7$ is shown in Fig. 12 where the base plane was restricted to the range $1.8 \leq \gamma / \nu \leq 2.2$ and $0 \leq \omega \leq 4$. The absolute minimum was found at $\gamma / \nu=1.952, \omega=2.24$, and a second, less pronounced minimum at $\gamma / \nu=2.112, \omega=0.01$. The results for the

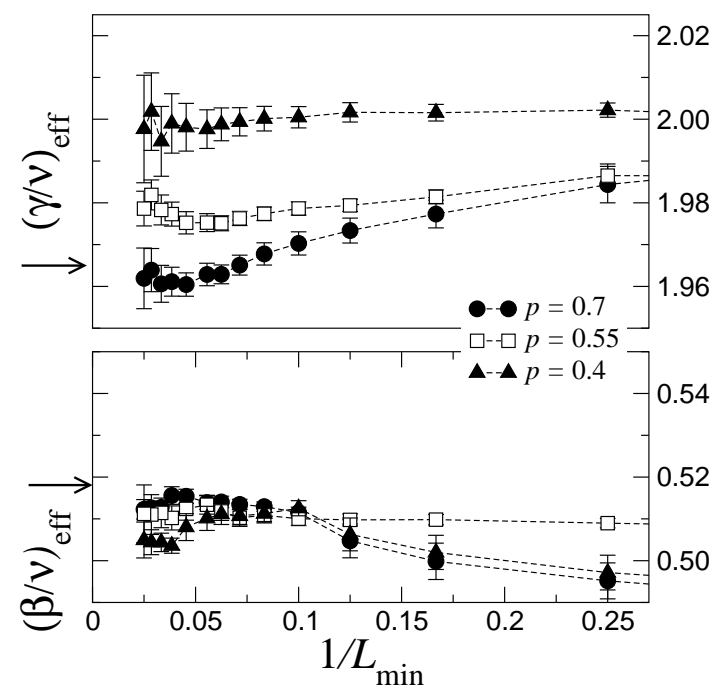

Fig. 11. Effective ratios $(\gamma / \nu)_{\text {eff }}$ and $(\beta / \nu)_{\text {eff }}$ as a function of $1 / L_{\min }$ for $p=0.7,0.55$, and 0.4 . The error bars correspond to the standard deviations of the power-law fits. The arrows indicate roughly the expected values for both the pure and disordered model. magnetisation respectively its logaritmic derivative and the other dilutions $p=0.55$ and 0.4 look qualitatively similar.

The figure may again be interpreted in favor of a competition between the two fixed points: the first one is characterized by a valley in the $\omega$-direction, with an absolute minimum for a large value of $\omega$, i.e., small corrections-toscaling since these corrections scale as $L^{-\omega}$. Then, this fixed point should be the disordered one and the second the percolation fixed point for which $\gamma / \nu \simeq 2.05$ and $\beta / \nu \simeq 0.48$ [66. But the accurate determination of the corrections-to-scaling exponent $\omega$ is very difficult because it strongly depends on the quantity considered $\left(\bar{\chi}_{\max }\right.$, $\bar{m}_{K_{\max }}$ or $\left.(d \ln \bar{m} / d K)_{K_{\max }}\right)$ and on the sizes included in the fits. Indeed, the valley in the $\omega$ direction does not display any deep minimum and the absolute minimum observed does not seem to be relevant concerning the value of $\omega$. We are thus not able to extract a numerical value of the corrections-to-scaling exponent, in contrast to previous claims that $\omega \approx 0.4$ [42,48, 49 .

\subsection{Temperature Scaling}

The temperature behaviour of the susceptibility and magnetisation should allow to avoid the previous difficulties generated by the vicinity of the ratios of the critical exponents. The critical exponents are indeed the following: pure Ising model: $\beta=0.3258(14), \gamma=1.2396(13)$ 62, site-diluted model: $\beta=0.3546(28), \gamma=1.342(10)$ [42.

As a function of the reduced temperature $t=\left(K_{c}-K\right)$ $(t<0$ in the low-temperature (LT) phase and $t>0$ in the high-temperature (HT) phase) and the system size $L$, the magnetisation and the susceptibility are expected to scale

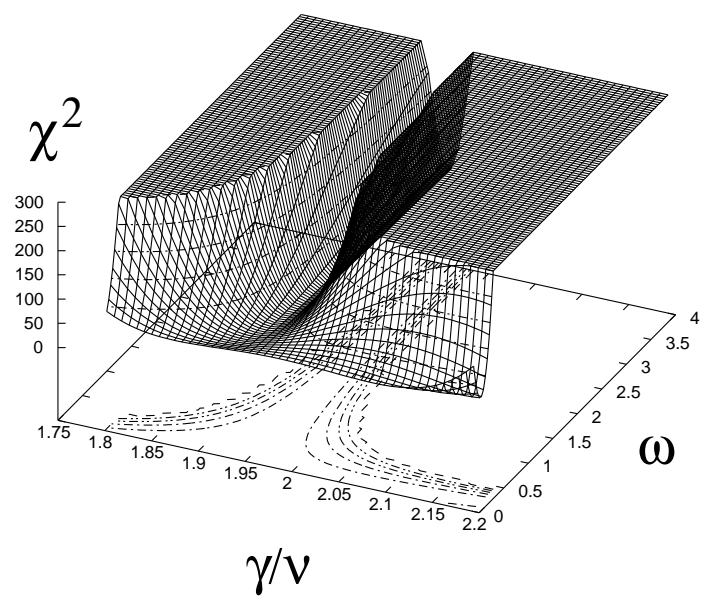

Fig. 12. Plot of the $\chi^{2}$-landscape deduced from linear fits of $\bar{\chi}_{\max }(L)=a_{\chi} L^{\gamma / \nu}\left(1+b_{\chi} L^{-\omega}\right)$ in the range $18 \leq L \leq 96$ for the bond-diluted Ising model with concentration $p=0.7$, exhibiting an absolute minimum at $\gamma / \nu=1.952, \omega=2.24$ and a secondary minimum at $\gamma / \nu=2.112, \omega=0.01$. The exponents are fixed parameters and the amplitudes are free. The cutoff at $\chi^{2}=300$ has been introduced in order to improve the clarity of the figure. 


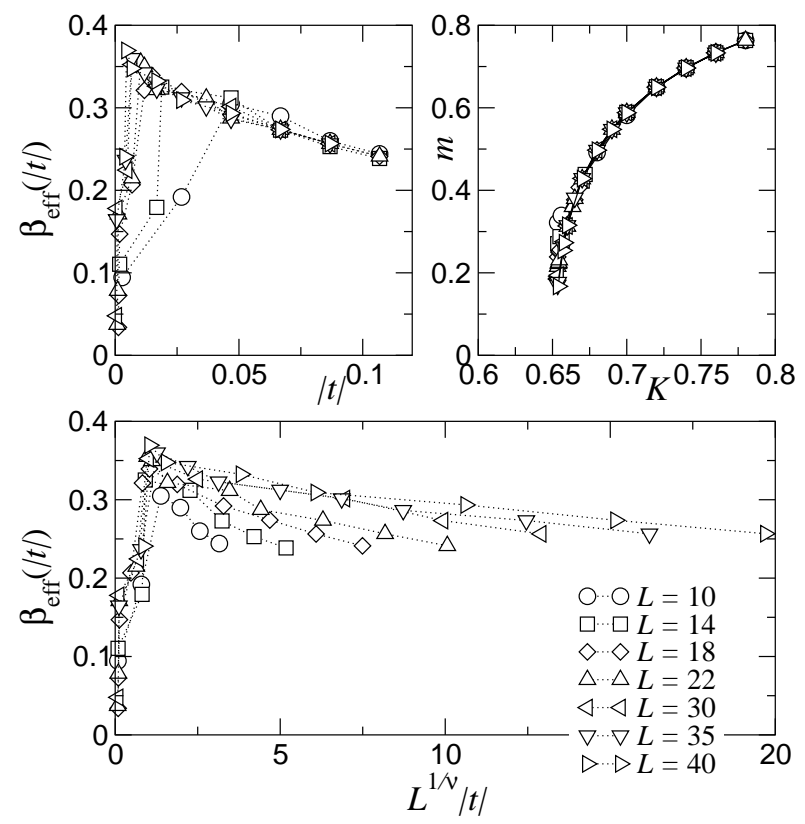

Fig. 13. Variation of the temperature dependent effective critical exponent $\beta_{\text {eff }}(|t|)=d \ln \bar{m} / d \ln |t|$ (in the low-temperature phase) as a function of the reduced temperature $|t|$ (top) and $L^{1 / \nu}|t|$ (bottom) for the bond-diluted Ising model with $p=0.7$ and several lattice sizes $L$. The data points are directly obtained from Monte Carlo data (no histogram reweighting). The magnetisation vs. the coupling strength $K=J / k_{B} T$ in the ordered phase $K>K_{c}^{\infty}$ is shown in the upper part.

as:

$$
\begin{aligned}
& \bar{m}(t, L) \sim|t|^{\beta} f_{ \pm}\left(L^{1 / \nu}|t|\right), \\
& \bar{\chi}(t, L) \sim|t|^{-\gamma} g_{ \pm}\left(L^{1 / \nu}|t|\right),
\end{aligned}
$$

where $f_{ \pm}$and $g_{ \pm}$are scaling functions of the variable $x=L^{1 / \nu}|t|$ and the subscript \pm stands for the HT/LT phases. Then we can define temperature dependent effective critical exponents

$$
\begin{aligned}
& \beta_{\text {eff }}(|t|)=d \ln \bar{m} / d \ln |t|, \\
& \gamma_{\text {eff }}(|t|)=-d \ln \bar{\chi} / d \ln |t|,
\end{aligned}
$$

which should converge towards the asymptotic critical exponents $\beta$ and $\gamma$ when $L \rightarrow \infty$ and $|t| \rightarrow 0$. The results for $p=0.7$ are shown in Figs. 13 and 14

The effective exponents $\beta_{\text {eff }}(|t|)$ evolve as a function of $|t|$ between a maximum value and 0 in the two $|t|$ directions: when $|t|$ is large ( $K$ far from $K_{c}$ ), the system is outside the critical region and the description with the critical exponents is no more valid whereas when $|t| \rightarrow 0$, the correlation length becomes of the same order as the linear size of the system and the finite-size effects become very strong. Nevertheless, just before the curves bend down, for the largest lattice sizes we can read off that $\beta_{\text {eff }}(|t|) \approx 0.34-0.36$. In the case of the susceptibility, for the greatest sizes, $\gamma_{\text {eff }}(|t|)$ is stable around 1.34 when $|t|$ is not too small, i.e., when the finite-size effects are not too strong. The plot of $\gamma_{\text {eff }}(|t|)$ vs. the rescaled variable

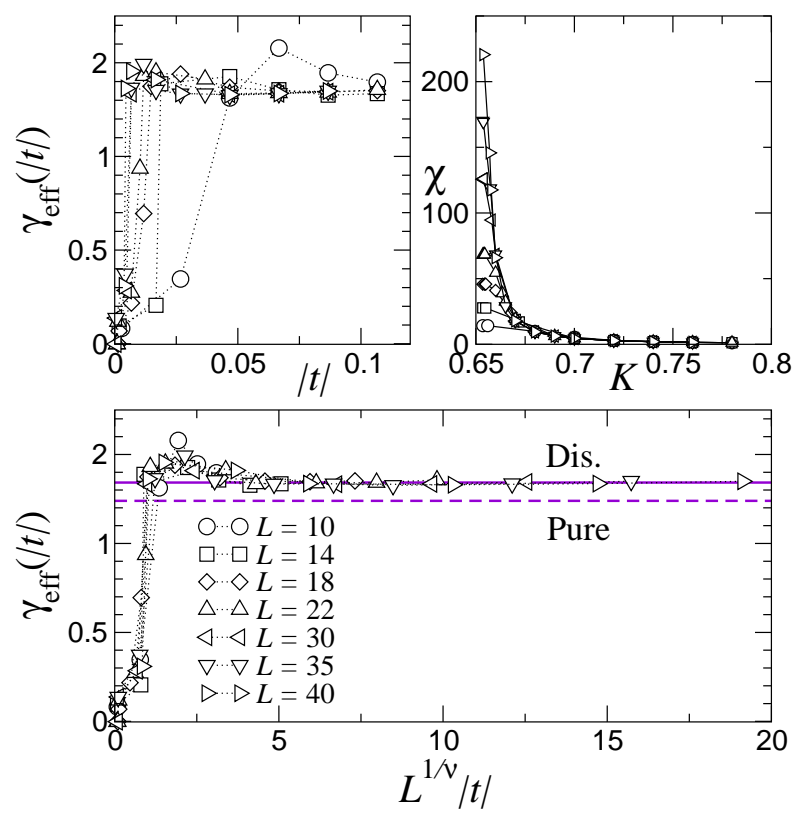

Fig. 14. Variation of the temperature dependent effective critical exponent $\gamma_{\text {eff }}(|t|)=-d \ln \bar{\chi} / d \ln |t|$ (in the low-temperature phase) as a function of the reduced temperature $|t|$ (top) and $L^{1 / \nu}|t|$ (bottom) for the bond-diluted Ising model with $p=0.7$ and several lattice sizes $L$. The horizontal dashed lines indicate the pure and site-diluted values of $\gamma$. The susceptibity vs. the coupling strength $K=J / k_{B} T$ in the ordered phase is shown in the upper part.

$L^{1 / \nu}|t|$ shows that the critical power-law behaviour holds in different temperature ranges for the different sizes studied. As expected, the size effects are more sensitive when the lattice size is small and the critical behaviour is better described when the size increases.

A more quantitative analysis is possible for the concentrations $p=0.55$ and 0.7 for which the most accurate temperature simulations have been done. ¿From the temperature behaviour of the susceptibility, we have directly extracted the power-law exponent $\gamma$ from error weighted least-squares fits by choosing the temperature range that gives the smallest $\chi^{2} /$ d.o.f for several system sizes. The results are given in Table 4 We see that all estimates are consistent with $\gamma \approx 1.34-1.36$, which is clearly different from the pure model's exponent of $\gamma \approx 1.24$. In particular we do not observe in this analysis any pronunced differences between the two dilutions. For $p=0.4$, our main dilution closest to the percolation threshold, the number of accurate temperature points is unfortunately not large enough to allow for reliable fits.

From the previous expressions of the magnetisation and susceptibility as a function of the reduced temperature and size, following a procedure proposed by Binder and Landau [72 a long time ago, it is instructive to plot the scaling functions $f_{ \pm}(x)$ and $g_{ \pm}(x)$. For finite size and $|t| \neq 0$, the scaling functions may be Taylor expanded in powers of the inverse scaling variable $x^{-1}=\left(L^{1 / \nu}|t|\right)^{-1}$, for example in the case of the susceptibility $\bar{\chi}_{ \pm}(t, L)=$ 

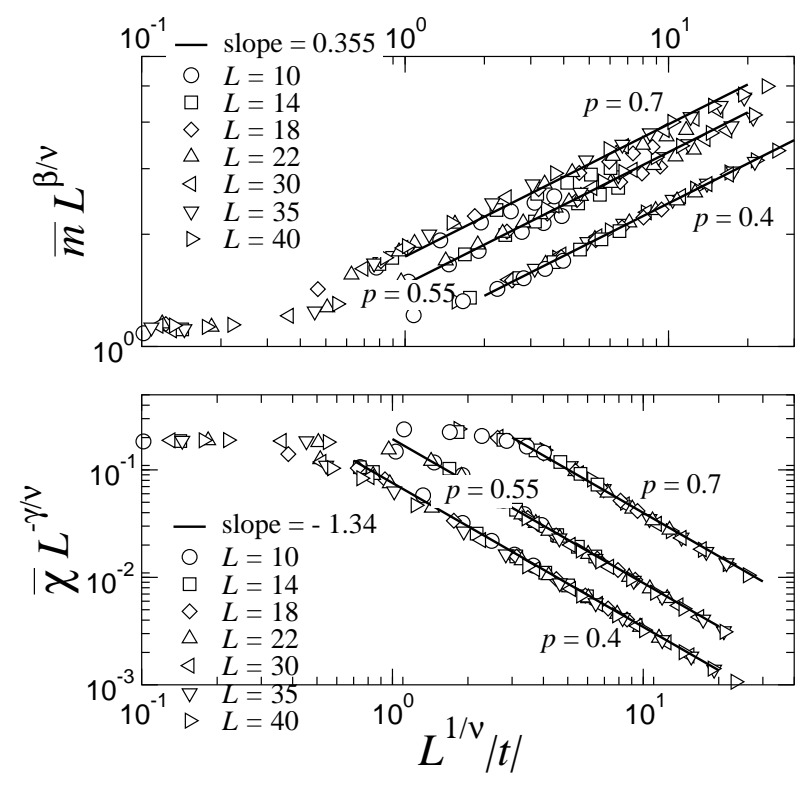

Fig. 15. Log-log plot of the scaling functions $\bar{m} L^{\beta / \nu}$ (top) and $\bar{\chi} L^{-\gamma / \nu}$ (bottom) against $L^{1 / \nu}|t|$ for $p=0.4,0.55$, and 0.7 in the low-temperature ordered phase $\left(K>K_{c}^{\infty}\right)$. The lines show the power-law behaviours with the exponents $\beta \simeq 0.355$ and $\gamma \simeq 1.34$ which characterize the disorder fixed point. The data for the smallest values of $L^{1 / \nu}|t|$ which do not lye on the master curve show the size effects described previously when the correlation length $\xi$ is limited by the linear size $L$.

Table 4. Critical exponent $\gamma$ deduced from log-log fits of the susceptibility vs. the reduced temperature $|t|$ in the ordered phase for the concentrations $p=0.55$ and 0.7 .

\begin{tabular}{llllll}
\hline$p=0.55$ & $L$ & 40 & & 22 & \\
\hline & $\#$ points & 7 & & 12 & \\
& $\chi^{2} /$ d.o.f & 0.07 & & 1.6 & \\
& $\gamma$ & $1.36(3)$ & & $1.36(2)$ & \\
\hline$p=0.7$ & $L$ & 40 & 35 & 22 & 18 \\
\hline & $\#$ points & 10 & 8 & 9 & 8 \\
& $\chi^{2} /$ d.o.f & 8.37 & 0.01 & 0.08 & 0.81 \\
$\gamma$ & $1.32(1)$ & $1.34(5)$ & $1.40(4)$ & $1.34(5)$ \\
\hline
\end{tabular}

$|t|^{-\gamma}\left[g_{ \pm}(\infty)+x^{-1} g_{ \pm}^{\prime}(\infty)+O\left(x^{-2}\right)\right]$, where the amplitude $g_{ \pm}(\infty)$ is usually denoted by $\Gamma_{ \pm}$. Multiplying by $L^{-\gamma / \nu}$ leads to

$$
\bar{\chi}_{ \pm} L^{-\gamma / \nu}=\tilde{g}_{ \pm}(x)=\Gamma_{ \pm} x^{-\gamma}+O\left(x^{-\gamma-1}\right),
$$

where $\tilde{g}_{ \pm}(x)=x^{-\gamma} g_{ \pm}(x)$. The case of the magnetisation is slightly different, since the magnetisation is asymptotically vanishing in the high-temperature phase, and thus $\bar{m}_{ \pm}(t, L)=|t|^{\beta}\left[f_{ \pm}(\infty)+x^{-1} f_{ \pm}^{\prime}(\infty)+O\left(x^{-2}\right)\right]$ or

$$
\bar{m}_{ \pm} L^{\beta / \nu}=\tilde{f}_{ \pm}(x)=x^{\beta}\left\{\begin{array}{c}
0+B_{+}^{\prime} x^{-1}+O\left(x^{-2}\right) \\
B_{-}+B_{-}^{\prime} x^{-1}+O\left(x^{-2}\right)
\end{array}\right.
$$

which should give universal curves for the different sizes and temperatures.
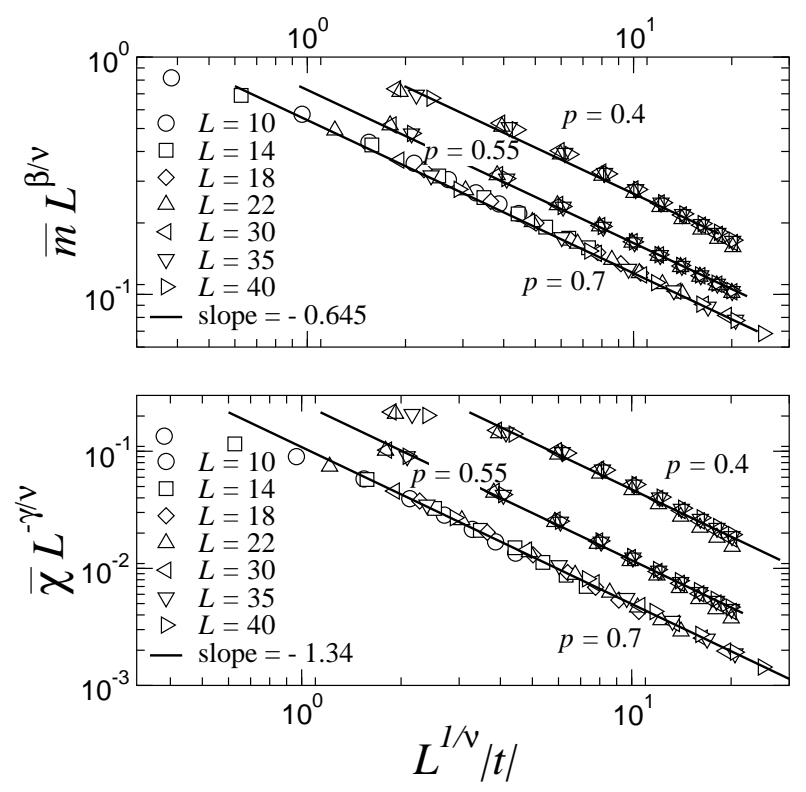

Fig. 16. Log-log plot of the scaling functions $\bar{m} L^{\beta / \nu}$ (top) and $\bar{\chi} L^{-\gamma / \nu}$ (bottom) against $L^{1 / \nu}|t|$ in the disordered phase $\left(K<K_{c}^{\infty}\right)$ for the three dilutions. The lines show the powerlaw behaviours with the exponents $\beta-1 \simeq-0.645$ and $-\gamma \simeq$ -1.34 which characterize the disorder fixed point.

The curves in the ordered phase shown in Fig. 15] are obviously universal master curves whose slopes, in a $\log$-log plot, give the critical exponents $\beta \simeq 0.355$ and $\gamma \simeq 1.34$. Indeed, when $|t| \rightarrow 0$ but with $L$ still larger than the correlation length $\xi$, one should recover the critical behaviour given by $\tilde{f}_{-}(x) \sim x^{\beta}$ and $\tilde{g}_{-}(x) \sim x^{-\gamma}$. The same procedure applied to the two other bond concentrations $p=0.55$ and 0.4 gives analogous results and confirms the universal values of the disordered critical exponents $\beta$ and $\gamma$, independent of the concentration as is illustrated in Fig. 15.

In the disordered phase $K<K_{c}^{\infty}, t>0$ the previous scaling assumptions for $\bar{m}$ and $\bar{\chi}$ lead to universal functions whose slopes on a logarithmic scale are equal to $\beta-1 \simeq-0.645$ and $-\gamma \simeq-1.34$, see Fig. [16]

Some combinations of critical amplitudes are also universal and thus characterize the critical point. In fact, critical amplitude ratios are often more sensitive to the universality class than the critical exponents themselves 73 . Among such ratios, $\Gamma_{+} / \Gamma_{-}$is directly accessible through our results. An example is illustrated in Fig. 17 where the scaling function of Eq. (11) is plotted against the scaling variable $x$. The values of the amplitudes which describe the approach to criticality from above and from below, $\Gamma_{+}$and $\Gamma_{-}$, respectively, are shown in shaded horizontal stripes. The ratios

$$
\begin{aligned}
& \Gamma_{+} / \Gamma_{-}=1.62 \pm 0.10(p=0.7), \\
& \Gamma_{+} / \Gamma_{-}=1.50 \pm 0.10(p=0.55), \\
& \Gamma_{+} / \Gamma_{-}=1.48 \pm 0.20(p=0.4)
\end{aligned}
$$

follow. The values obtained for the three dilutions are consistent within error bars but they unfortunately appear 


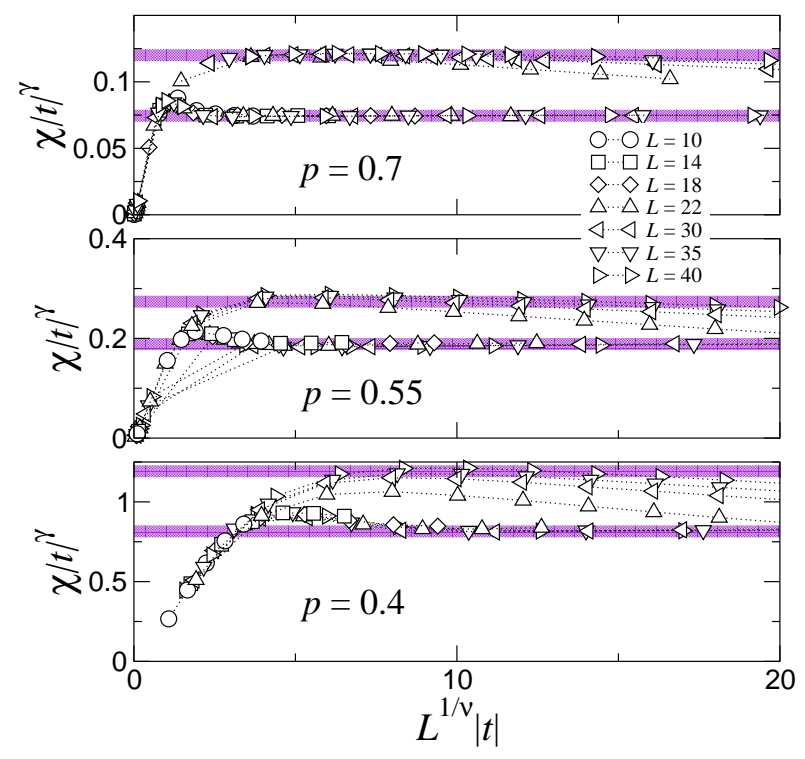

Fig. 17. Log-log plot of the scaling functions $\bar{\chi}|t|^{\gamma}$ against $L^{1 / \nu}|t|$ for the three dilutions. The shaded horizontal stripes indicate the critical amplitudes $\Gamma_{+}$and $\Gamma_{-}\left(<\Gamma_{+}\right)$, respectively.

to be in contradiction with a field-theoretic approach of Bervillier and Shpot [60, who predicted a ratio equal to $\Gamma_{+} / \Gamma_{-}=3.05(32)$. This is nevertheless not a crucially conflicting result, since susceptibility amplitudes of course depend on the definition used to compute the susceptibilities. At any rate, our estimates (16)-(18) for the disordered Ising model are clearly different from those for the pure model where $\Gamma_{+} / \Gamma_{-}$obtained with field-theoretic and high-temperature series expansion techniques varies between 4.70 and 4.95 [74, and a recent high-precision Monte Carlo study [75] concluded that $\Gamma_{+} / \Gamma_{-}=4.75(3)$.

\section{Conclusions}

In this paper, we have reported an intensive Monte Carlo study of the physical properties of the 3D disordered bonddiluted Ising model. As the critical exponents of the pure and disordered models are very close, the numerical procedure has to be carefully chosen, especially the thermal averaging and the average over the disorder realisations. We have determined numerically the phase diagram of the disordered model, in very good agreement with a singlebond effective-medium approximation.

The non-self-averaging at criticality, as discussed by Aharony and Harris, is confirmed here. At a quantitative level, the discrepancy between the numerical values of the normalized squared width of the susceptibility $R_{\chi}(L)$ in this work and that in Ref. 44] definitely comes from the difference in the definition of $\chi$. Although in our case $R_{\chi}(L)$ and to a lesser extent also $R_{m}(L)$ do not seem to approach a unique constant independent of the disorder concentration when the system size $L$ increases, we cannot exclude a unique limiting value when cross-over effects are properly taken into account. This possibility is corrobo-
Table 5. Critical exponents and critical amplitude ratio of the susceptibility for the different fixed points.

\begin{tabular}{llll}
\hline fixed point & $\nu$ & $\beta$ & $\gamma$ \\
\hline pure & $0.6304(13)$ & $0.3258(14)$ & $1.2396(13)$ \\
percolation & 0.89 & 0.40 & 1.82 \\
site-diluted & $0.6837(53)$ & $0.3546(28)$ & $1.342(10)$ \\
bond-diluted & $0.68(2)$ & $0.35(1)$ & $1.34(1)$ \\
\hline fixed point & $\beta / \nu$ & $\gamma / \nu$ & $\Gamma_{+} / \Gamma_{-}$ \\
\hline pure & $0.517(3)$ & $1.966(6)$ & $4.75(3)$ \\
percolation & 0.45 & 2.05 & \\
site-diluted & $0.519(3)$ & $1.963(5)$ & \\
bond-diluted & $0.515(5)$ & $1.97(2)$ & $1.50(20)$ \\
\hline
\end{tabular}

rated by our finite-size scaling study of critical exponents. A comparison of the underlying disorder distributions for the bond-diluted Ising and $q=4$ Potts models shows that the weight of rare events plays a much more crucial role for the critical properties of the latter model.

The main emphasis was on investigations of the critical behaviour of the disordered model based on a finitesize scaling study and on a complementary study of the temperature scaling behaviour. In the first case, we have shown the great influence of the cross-over phenomena between the pure, disorder and percolation fixed points which lead to the measurement of effective critical exponents dependent on the concentration of magnetic bonds. The temperature study, on the other hand, turned out to be much better behaved and allowed us to confirm the unique values of the critical exponents, independent of the concentration and in agreement with the site-diluted values, thus providing strong numerical evidence for the theoretically predicted universal behaviour of the two disorder distributions. These results are summarized in Table [5 A scaling study of the average of the maxima of the susceptibilities $\chi_{j}$ for each sample instead of the maximum of the average susceptibility would have been an interesting alternative. However, it is technically more complicated because from sample to sample the position of the maximum of the susceptibility varies and may be far from the simulation temperature making histogram reweighting unreliable. There should even appear samples with large bonddensity fluctuations where unconnected or weakly connected clusters of bonds coexist. Such special configurations may display a double-peaked (or more complicated) susceptibility curve resulting from the independence of these uncorrelated clusters.

Finally, we should remark that even though this work required a really huge amount of effort and CPU time, for some reasons that we are unable to identify, the accuracy of our results is not as good as in the work of Ballesteros et al. 24 for site-dilution. For example we were not able to conclude for a reliable estimate of the corrections-to-scaling exponent $\omega$ as they did. Nevertheless it is worth noticing that the temperature scaling is quite satisfying and led to the measurement of the suscep- 
tibility amplitude ratio $\Gamma_{+} / \Gamma_{-}$, a universal combination which was still unknown numerically, and which complements an estimate of other universal combinations of amplitudes reported recently in Refs. 45.76. As anticipated from a previous analysis for disordered systems, our estimates for the amplitude ratio $\Gamma_{+} / \Gamma_{-}$are clearly different from the pure model and thus yield the cleanest signal for a unique disorder fixed point.

The authors are happy to thank Yu. Holovatch and L.N. Shchur for many discussions. We also gratefully acknowledge financial support by the DAAD and A.P.A.P.E. through the PROCOPE exchange programme. This work was supported by the computer-time grants No. 2000007 of the Centre de Ressources Informatiques de Haute Normandie (CRIHAN), Rouen, No. 0620011 of CINES, Montpellier, No. hlz061 of NIC, Jülich, and No. h0611 of LRZ, München. W.J. also acknowledges partial support by the EU-Network HPRN-CT-1999-000161 "Discrete Random Geometries: From Solid State Physics to Quantum Gravity" and the German-Israel Foundation (GIF) under grant No. I-653-181.14/1999.

\section{References}

1. J.L. Cardy, Scaling and Renormalization in Statistical Physics (Cambridge University Press, Cambridge, 1996), chap. 8.

2. In this paper, we do not discuss the case of long-range correlated disorder (e.g., the McCoy-Wu model or quantum chains) which may lead to an infinite-randomness disorder fixed point under renormalization (see, e.g., R. Sknepnek and T. Vojta, e-print cond-mat/0311394 and references therein).

3. A.B. Harris, J. Phys. C 7, 1671 (1974).

4. B.N. Shalaev, Phys. Rep. 237, 129 (1994)

5. L. Schwenger, K. Budde, C. Voges, H. Pfnür, Phys. Rev. Lett. 73, 296 (1994).

6. C. Voges, H. Pfnür, Phys. Rev. B 57, 3345 (1998).

7. Y. Imry, M. Wortis, Phys. Rev. B 19, 3580 (1979).

8. M. Aizenman, J. Wehr, Phys. Rev. Lett. 62, 2503 (1989).

9. K. Hui, A.N. Berker, Phys. Rev. Lett. 62, 2507 (1989).

10. F.Y. Wu, Rev. Mod. Phys. 54, 235 (1982).

11. B. Berche, C. Chatelain, to appear in Order, Disorder, and Criticality, edited by Yu. Holovatch (World Scientific, Singapore, 2004) (in print), e-print cond-mat/0207421

12. A.W.W. Ludwig, Nucl. Phys. B 285 [FS19], 97 (1987).

13. S. Chen, A.M. Ferrenberg, D.P. Landau, Phys. Rev. Lett. 169, 1213 (1992); Phys. Rev. E 52, 1377 (1995).

14. Vl. Dotsenko, M. Picco, P. Pujol, Nucl. Phys. B 455 [FS], 701 (1995).

15. G. Jug, B.N. Shalaev, Phys. Rev. B 54, 3442 (1996).

16. J.L. Cardy, J.L. Jacobsen, Phys. Rev. Lett. 79, 4063 (1997).

17. M. Picco, Phys. Rev. Lett. 79, 2998 (1997).

18. J.L. Jacobsen, J.L. Cardy, Nucl. Phys. B 515, 701 (1998).

19. C. Chatelain, B. Berche, Phys. Rev. Lett. 80, 1670 (1998).

20. A. Roder, J. Adler, W. Janke, Phys. Rev. Lett. 80, 4697 (1998); Physica A 265, 28 (1999).

21. T. Olson, A.P. Young, Phys. Rev. B 60, 3428 (1999).

22. C. Chatelain, B. Berche, Nucl. Phys. B 572, 626 (2000).
23. W. Janke, R. Villanova, Nucl. Phys. B 489, 679 (1997).

24. H.G. Ballesteros, L.A. Fernández, V. Martín-Mayor, A. Muñoz Sudupe, G. Parisi, J.J. Ruiz-Lorenzo, Phys. Rev. B 61, 3215 (2000).

25. W. Janke and S. Kappler, unpublished (1996).

26. C. Chatelain, B. Berche, W. Janke, P.E. Berche, Phys. Rev. E, 64, 036120 (2001).

27. C. Chatelain, P.E. Berche, B. Berche, W. Janke, Comp. Phys. Comm. 147, 431 (2002).

28. C. Chatelain, P.E. Berche, B. Berche, W. Janke, Nucl. Phys. B (Proc. Suppl.) 106\&107, 899 (2002).

29. P.E. Berche, C. Chatelain, B. Berche, W. Janke, in: High Performance Computing in Science and Engineering, edited by S. Wagner, W. Hanke, A. Bode, F. Durst (Springer, Berlin, 2003), p. 227.

30. W. Janke, P.E. Berche, C. Chatelain, B. Berche, Leipzig preprint (April 2003), e-print cond-mat/0304642 to appear in: Computer Simulation Studies in CondensedMatter Physics XVI, edited by D.P. Landau, S.P. Lewis, H.-B. Schüttler (Springer, Berlin, 2004) (in print).

31. D.P. Landau, Phys. Rev. B 22, 2450 (1980).

32. J. Marro, A. Labarto, J. Tejada, Phys. Rev. B 34, 347 (1986).

33. D. Chowdhury, D. Staufer, J. Stat. Phys. 44, 203 (1986).

34. P. Braun, M. Fähnle, J. Stat. Phys. 52, 775 (1988).

35. J.S. Wang, D. Chowdhury, J. Phys. France 50, 2905 (1989).

36. J.S. Wang, M. Wöhlert, H. Mühlenbein, D. Chowdhury, Physica A 166, 173 (1990).

37. T. Holey, M. Fähnle, Phys. Rev. B 41, 11709 (1990).

38. H.O. Heuer, Europhys. Lett. 12, 551 (1990).

39. H.O. Heuer, Phys. Rev. B 42, 6746 (1990)

40. H.O. Heuer, J. Phys. A 26, L333 (1993).

41. M. Hennecke, Phys. Rev. B 48, 6271 (1993).

42. H.G. Ballesteros, L.A. Fernández, V. Martín-Mayor, A. Muñoz Sudupe, G. Parisi, J.J. Ruiz-Lorenzo, Phys. Rev. B 58, 2740 (1998).

43. S. Wiseman, E. Domany, Phys. Rev. Lett. 81, 22 (1998).

44. S. Wiseman, E. Domany, Phys. Rev. E 58, 2938 (1998).

45. P. Calabrese, V. Martín-Mayor, A. Pelissetto, E. Vicari, e-print cond-mat/0306272

46. Y. Holovatch, T. Yavors'kii, J. Stat. Phys. 92, 785 (1998).

47. R. Folk, Y. Holovatch, T. Yavors'kii, J. Phys. Stud. (Ukraine) 2, 213 (1998).

48. R. Folk, Y. Holovatch, T. Yavors'kii, JETP Lett. 69, 747 1999).

49. R. Folk, Y. Holovatch, T. Yavors'kii, Phys. Rev. B 61, 15114 (2000).

50. R. Folk, Y. Holovatch, T. Yavors'kii, Physics Uspiekhi 173, 175 (2003) [e-print cond-mat/0106468.

51. K.B. Varnashev, Phys. Rev. B 61, 14660 (2000).

52. D.V. Pakhnin, A.I. Sokolov, Phys. Rev. B 61, 15130 (2000).

53. D.V. Pakhnin, A.I. Sokolov, JETP Lett. 71, 412 (2000).

54. A. Pelissetto, E. Vicari, Phys. Rev. B 62, 6393 (2000).

55. P. Calabrese, P. Parrucini, A. Pelissetto, E. Vicari, e-print cond-mat/0307699

56. K.E. Newman, E.K. Riedel, Phys. Rev. B 25, 264 (1982).

57. J. Jug, Phys. Rev. B 27, 609 (1983).

58. I.O. Mayer, J. Phys. A 22, 2815 (1989).

59. I.O. Mayer, A.I. Sokolov, B.N. Shalaev, Ferroelectrics 95, 93 (1989).

60. C. Bervillier, M. Shpot, Phys. Rev. B 46, 955 (1992). 
61. A. Aharony, A.B. Harris, S. Wiseman, Phys. Rev. Lett. 81, 252 (1998).

62. R. Guida, J. Zinn-Justin, J. Phys. A 31, 8103 (1998).

63. R.H. Swendsen, J.S. Wang, Phys. Rev. Lett. 58, 86 (1987).

64. A.M. Ferrenberg, R.H. Swendsen, Phys. Rev. Lett. 61, 2635 (1988).

65. A.M. Ferrenberg, R.H. Swendsen, Phys. Rev. Lett. 63, 1195 (1989).

66. C.D. Lorenz, R.M. Ziff, Phys. Rev. E 57, 230 (1998).

67. P.E. Berche, C. Chatelain, B. Berche, W. Janke, Comp. Phys. Comm. 147, 427 (2002).

68. A.L. Talapov, H.W.J. Blöte, J. Phys. A 29, 5727 (1996).

69. L. Turban, Phys. Lett. A 75, 307 (1980).

70. M. Hellmund, W. Janke, Comp. Phys. Comm. 147, 435 (2002).

71. A. Aharony, A.B. Harris, Phys. Rev. Lett. 77, 3700 (1996).

72. K. Binder, D.P. Landau, Phys. Rev. B 21, 1941 (1980).

73. L.N. Shchur, O.A. Vasilyev, Phys. Rev. E 65, 016107 (2002).

74. For a list of results and references to original work, see Ref. [5].

75. M. Caselle, M. Hasenbusch, J. Phys. A 30, 4963 (1997).

76. P. Calabrese, M. De Prato, A. Pelissetto, E. Vicari, e-print cond-mat/0305434 